\title{
Reused Lead
}

National Cancer Institute

\section{Source}

National Cancer Institute. Reused Lead. NCI Thesaurus. Code C102284.

The lead has been left in situ and reused. 